\title{
Atypical patterns in portable monitoring for sleep apnoea: features of nocturnal epilepsy?
}

\author{
Liborio Parrino*, Giulia Milioli*, Andrea Grassi*, Fernando De Paolis*, \\ Silvia Riccardi*, Elena Colizzi*, Marcello Bosii ${ }^{*}$ and Mario Giovanni Terzano*
}

ABSTRACT: Atypical cardiorespiratory patterns can be found during routine clinical use of portable monitoring for diagnosis of sleep-disordered breathing (SDB).

Over 1,000 consecutive portable recordings were analysed to study the potential ictal nature of stereotyped cardiorespiratory and motor patterns. Snoring, airflow, thoracic effort, pulse rate, body position, oxygen saturation and activity of the anterior tibialis muscles were quantified. Recordings showing stereotyped polygraphic patterns recurring throughout the night, but without the features of sleep apnoea (apnoea/hypopnoea index $<5$ events $\cdot h^{-1}$ ), were selected for investigation. Once included in the study, patients underwent attended nocturnal video polysomnography.

A total of 15 recordings showing repeated polygraphic patterns characterised by a sequence of microphone activation, respiratory activity atypical for sleep and wakefulness, heart rate acceleration and limb movements, followed by body position change, were selected for investigation. Once included in the study, patients underwent attended nocturnal video polysomnography that showed frontal epileptic discharges triggering periodic electroencephalographic arousals, autonomic activation and stereotyped motor patterns. A diagnosis of nocturnal frontal lobe epilepsy (NFLE) was established for all patients.

NFLE should be taken into consideration in patients with stereotyped and recurrent behavioural features during portable monitoring carried out for diagnosis of SDB.

KEYWORDS: Sleep apnoea diagnosis and treatment, sleep-disordered breathing, sleep disorders, sleep medicine

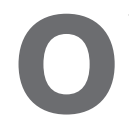
bstructive sleep apnoea syndrome (OSAS) is a sleep disorder characterised by recurrent episodes of complete (apnoea) or partial (hypopnoea) upper airway occlusion occurring during sleep [1]. Respiratory events are typically terminated by brief arousals, which result in disruption of normal sleep architecture and are often coupled with tachycardia, leg jerks or complex somatomotory behaviour such as sleeptalking or brisk movements involving the arms, trunk and neck. Patients with OSAS are usually unaware of these behavioural alterations, which are typically reported by bed partners. Snorers with choking gasps often report nocturnal awakenings accompanied by the need to sit up abruptly in the bed to recover regular breathing. In addition, diurnal symptoms such as non-restorative sleep, fatigue or excessive sleepiness are common complaints.

Similar findings can be described in patients with other sleep disorders, in particular those affected by nocturnal frontal lobe epilepsy (NFLE) [1]. NFLE is partial epilepsy in which electroencephalographic (EEG) abnormalities and clinical manifestations appear almost exclusively during sleep. Motor activity comprises distinct paroxysmal sleeprelated attacks of variable duration and behavioural complexity: minor motor events (MMEs) represented by short-lasting (2-4 s) stereotyped movements, involving the limbs, the axial musculature and/or the head; paroxysmal arousals (PAs), which consist of short attacks (lasting 5-10 s), characterised by sudden arousal often accompanied by dystonic and dyskinetic features; and major attacks (MAs) characterised by stereotyped movements, such as asymmetric tonic or dystonic posturing or other bizarre behaviours, lasting 20-30 s. Moreover, some patients can also manifest deambulatory behaviour during seizures [24]. The motor patterns are usually stereotyped and recur several times per night, every night. During seizures, polysomnography (PSG) shows transient autonomic alterations in cardiac rhythm and
AFFILIATIONS

*Sleep Disorders Center, Dept of Neurosciences, University of Parma, Parma, and

\#Pulmonary Operative Unit, Dept of Thoracic Diseases, G.B. Morgagni-L. Pierantoni Hospital, Forlì, Italy.

CORRESPONDENCE

L. Parrino

Centro di Medicina del Sonno,

Sezione di Neurologia, Dipartimento di Neuroscienze

Azienda Ospedaliera Universitaria

Via Gramsci 14

43100 Parma

Italy

E-mail: liborio.parrino@unipr.it

Received:

Nov 182011

Accepted after revision:

April 282012

First published online:

June 142012 
respiratory activity. Several patients with NFLE report poor sleep quality, daytime fatigue or sleepiness due to an increase of arousal instability and sleep fragmentation [5]. Because of the absence of specific diagnostic criteria, the diagnosis of NFLE relies principally on clinical history and on the recording of nocturnal seizures with semeiological features ranging from MMEs to PAs and MAs.

While a number of parasomnias (confusional arousals, sleep terrors and sleepwalking) tend to disappear or decrease in frequency after adolescence, awakenings with the feeling of being choked, sleep-talking, complex motor activity during sleep and excessive daytime sleepiness are common clinical features in adults with OSAS or NFLE. These disorders can coexist and potentially exacerbate each other. Moreover, due to overlapping clinical features, NFLE can be misdiagnosed as OSAS [6].

PSG is the gold standard for detecting respiratory events in OSAS but in Italy the diagnosis of sleep-disordered breathing (SDB) in adults is often documented using portable monitoring without EEG channels. Thus, abnormal nocturnal symptoms cannot be correlated with the presence of epileptiform discharges (EDs) and paroxysmal motors attacks cannot be documented by video recording procedures.

The present study aimed to evaluate the potential ictal nature of stereotyped cardiorespiratory and motor patterns in patients investigated for SDB.

\section{MATERIALS AND METHODS}

Over 1,000 consecutive portable recordings of patients who were referred to our Sleep Disorders Center for SDB (University of Parma, Parma, Italy), carried out over 15 months, were analysed. Level 3 sleep tests (table 1) [7], based on snoring, airflow, thoracic and abdominal effort, pulse rate, body position, oxygen saturation, and the activity of the anterior tibialis muscles were carried out according to the American Academy of Sleep Medicine (AASM) practice parameters [8]. Only recordings showing repeated, time-limited, stereotyped polygraphic patterns recurring throughout the night, but with an apnoea/hypopnoea index (AHI) $<5$ events $\cdot \mathrm{h}^{-1}$, were selected for investigation. No respiratory patterns analysed met the sleep breathing flow characteristics for detection of wakefulness in patients with OSAS $[9,10]$. Periodic limb movement (PLM) disorder was diagnosed for a PLM index $\geqslant 15$ events $\cdot h^{-1}[1]$.
Once included in the study, patients underwent an attended nocturnal video PSG. Sleep was recorded from F4-M1, C4-M1, O2-M1 derivations integrated by bipolar montages (Fp1-F3, F3C3, C3-P3, P3-O1 and Fp2-F4, F4-C4, C4-P4, P4-O2) used to optimise the scoring of cyclic alternating pattern (CAP). PSG measures included electro-oculographic channels, submental electromyogram, thoracic and abdominal effort, oxygen saturation, activity of the anterior tibialis muscles, and pulse rate. Analysis of conventional sleep variables was based on the AASM rules [8], while CAP parameters were measured according to the CAP atlas [11].

A questionnaire to quantify sleepiness was submitted to all patients [12].

\section{RESULTS}

A total of 15 level-3 portable recordings (table 1), carried out for diagnosis of SDB, met the inclusive criteria. Recordings were taken of eight males and seven females (mean age 35 (range 2743) yrs) who had a body mass index of $26.7 \pm 4.4 \mathrm{~kg} \cdot \mathrm{m}^{-2}$. The selected patients had complained of $\geqslant 6$ months of irregular snoring, sudden nocturnal awakenings associated with respiratory pauses, choking gasps or tachycardia. Sitting up abruptly in the bed, sleep-related limb movements and sleep-talking were often reported. Poor sleep quality and excessive daytime sleepiness were common findings. Evidence of relevant upper airway narrowing was found in seven out of 15 patients. No patient had a history of daytime epileptic seizures (table 2).

Portable monitoring excluded the diagnosis of sleep apnoea in all patients, whereas nocturnal PLM disorder was diagnosed in four patients (table 3 ).

All selected recordings showed an average of six polygraphic patterns characterised by motor and autonomic features (mean duration $4 \pm 1.5 \mathrm{~min}$ ). In particular, sequences of respiratory activity atypical for sleep and wakefulness, heart rate acceleration and limb movements, followed by body position change, recurred, stereotyped in each patient, throughout the night (fig. 1 and fig. 2). These patterns were always accompanied by an intense, sudden and transient activation of the microphone, similar to a vocalisation. There were no episodes of snoring before and after these events and the sequence was never accompanied by a desaturation.

In nocturnal video PSG recordings, frontal EDs triggered periodic EEG arousals often correlated with autonomic activation

TABLE 1 Classication of levels of studies of sleep apnoea evaluation

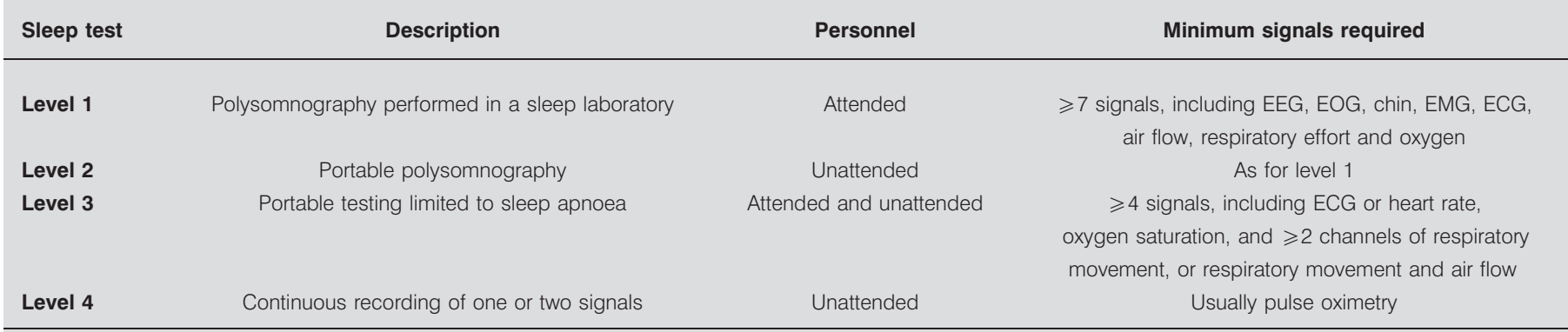

EEG: electroencephalography; EOG: electro-oculography; chin: submental electromyogram; EMG: electromyography. Information from [7]. 
TABLE 2 Clinical features of 15 selected patients

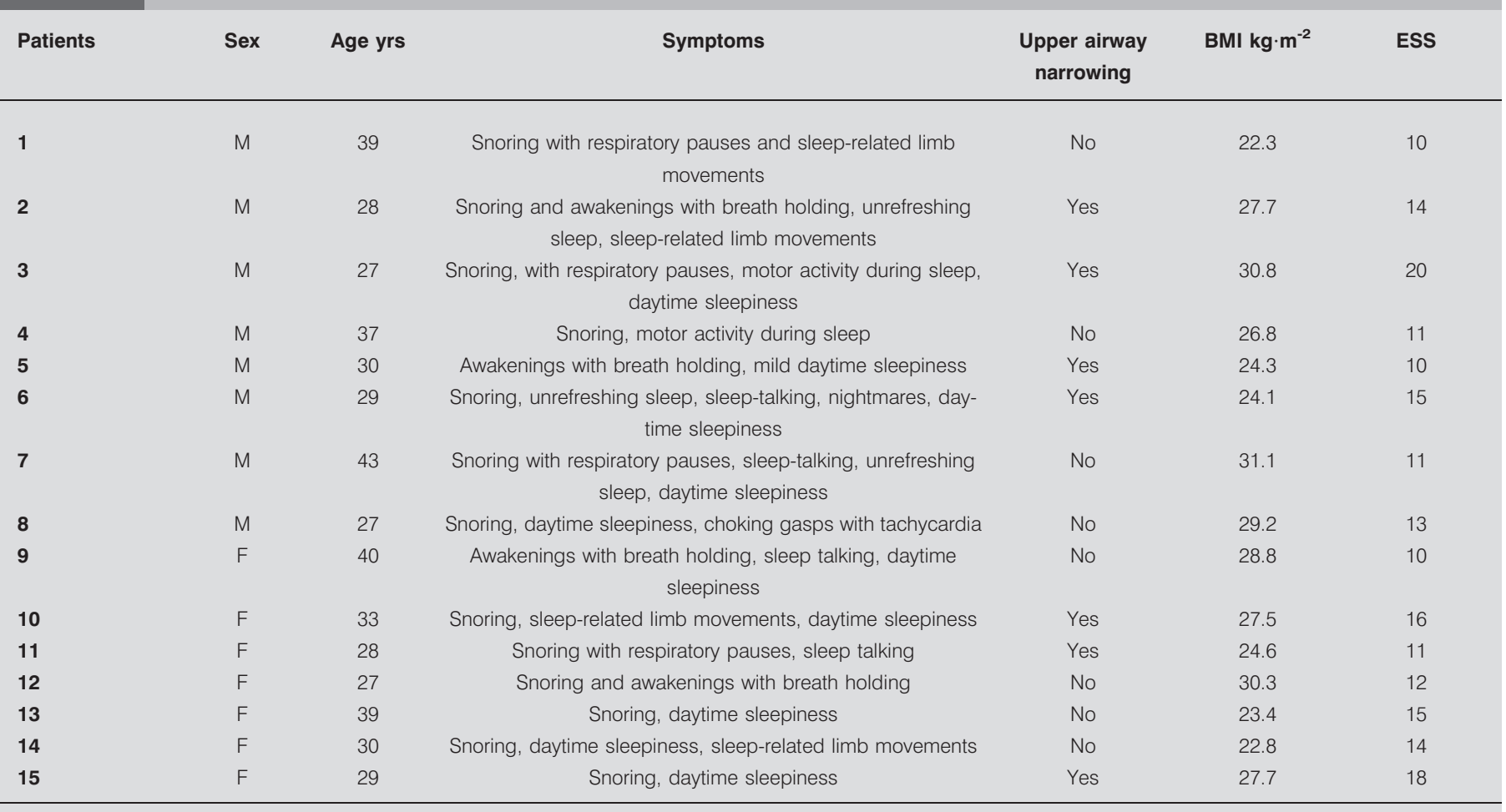

Data are presented as n, unless otherwise stated. BMI: body mass index; ESS: Epworth Sleepiness Scale [12]; M: male; F: female.

TABLE 3 Cardiorespiratory and video-polysomnographic parameters of the 15 selected patients

\begin{tabular}{|c|c|c|c|c|c|c|c|c|c|}
\hline \multirow[t]{2}{*}{ Patients } & \multicolumn{2}{|c|}{ Cardiorespiratory monitoring } & \multicolumn{7}{|c|}{ Nocturnal video polysomnography } \\
\hline & $A H I$ events $\cdot h^{-1}$ & $\begin{array}{l}\text { PLM index } \\
\text { events } \cdot h^{-1}\end{array}$ & SE \% & $\mathrm{SL} \min$ & $\mathrm{N} 1+\mathrm{N} 2 \%$ & N3 \% & REM \% & CAP rate $\%$ & Motor attacks \\
\hline 1 & 3.1 & 11.3 & 85.6 & 52.5 & 62.1 & 14.5 & 23.4 & 69.0 & 5 MMEs \\
\hline 2 & 2.5 & 20 & 88.7 & 5.0 & 51.8 & 21.3 & 26.9 & 56.2 & 10 MMEs, 2 MAs \\
\hline 5 & 3.8 & 3.3 & 98.8 & 43.0 & 54.1 & 17.5 & 28.4 & 81.8 & $1 \mathrm{MA}$ \\
\hline 6 & 3.1 & 2 & 97.6 & 11.2 & 28 & 52.1 & 19.9 & 70.7 & $1 \mathrm{MA}$ \\
\hline 7 & 4.0 & 10.6 & 85.5 & 6.7 & 52.4 & 31.9 & 15.7 & 55.6 & 5 MMEs \\
\hline 8 & 2.5 & 19.9 & 85.3 & 5.0 & 50.4 & 12.3 & 34.3 & 62.1 & 2 MMEs, 1 PA \\
\hline 9 & 3.3 & 15.4 & 72.0 & 30.5 & 34.3 & 31.4 & 34.3 & 70.3 & 2 PAs, $1 \mathrm{MA}$ \\
\hline 10 & 3.6 & 4.3 & 98.7 & 20.3 & 25.2 & 26.5 & 48.3 & 75.2 & 4 MMEs, $1 \mathrm{MA}$ \\
\hline 15 & 3.7 & 4.5 & 83.5 & 12.4 & 57.0 & 24.4 & 18.6 & 47.9 & 3 MMEs, 1 MA \\
\hline
\end{tabular}

Data are presented as n, unless otherwise stated. AHI: apnoea/hypopnoea index; PLM: periodic limb movements; SE: sleep efficiency; SL: sleep latency; N1+N2: nonrapid eye movement (NREM) stage 1 and 2; N3: NREM sleep stage 3; REM: rapid eye movement sleep; CAP: cyclic alternating pattern; MME: minor motor event [2]; MA: major attack [3]; PA: paroxysmal arousal [4].

FIGURE 1. (Following page.) Polygraphic stereotyped pattern, recurring throughout the night, characterised by intense, sudden and transient activation of the microphone, similar to a vocalisation; respiratory activity atypical for sleep and wakefulness; heart rate acceleration and limb movements; followed by body position change (boxed). The figure represents $1 \mathrm{~h}$ of sleep. Micro: microphone; Flow: airflow; Tor: thoracic effort; $\mathrm{Sp}_{\mathrm{O}} \mathrm{O}_{2}$ : arterial oxygen saturation measured by pulse oximetry; fc: cardiac frequency; Pos: body position (E: upright; P: prone; P: supine; D: right; S: left), PLMs: activity of the anterior tibialis muscles. 


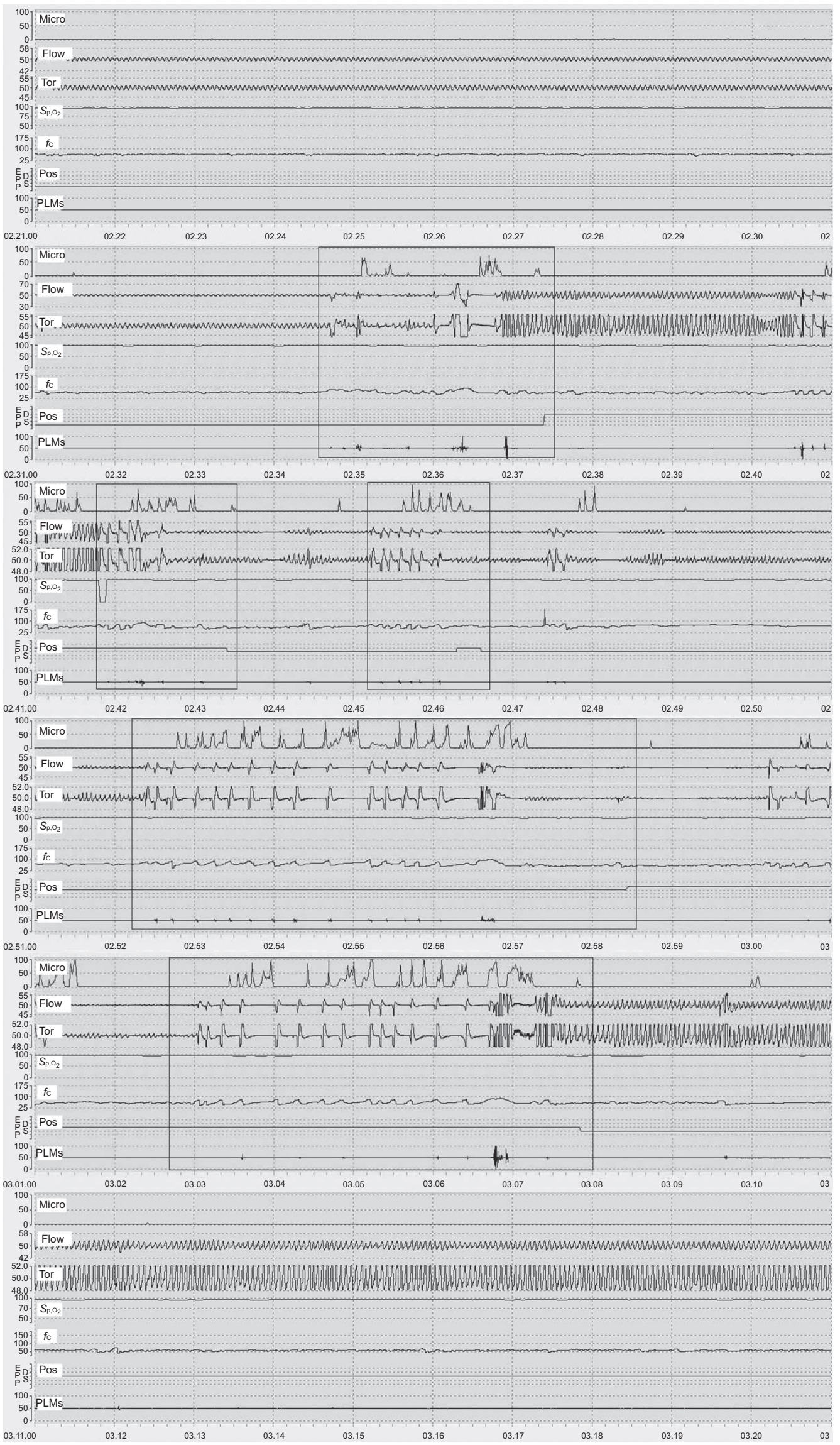



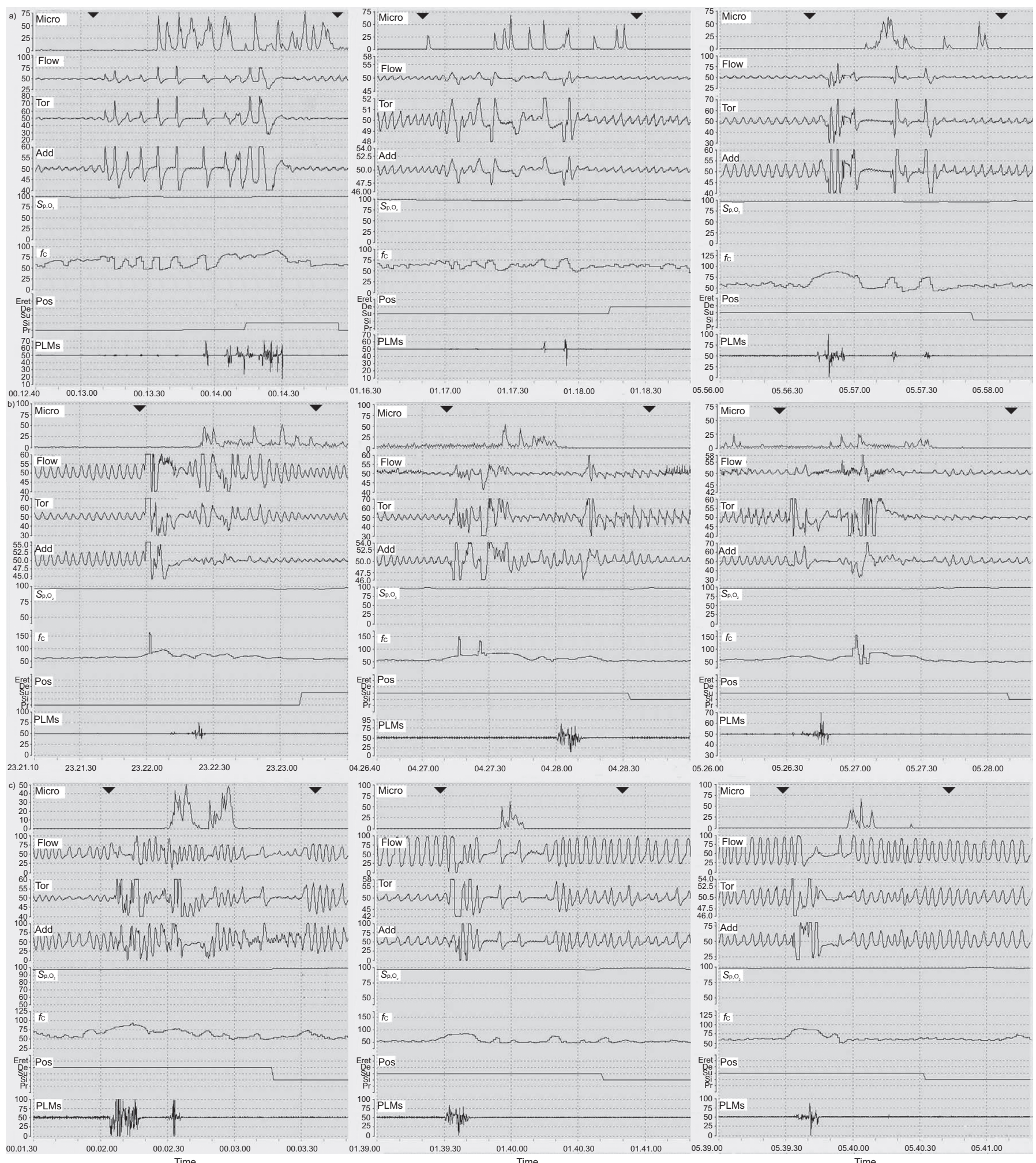

FIGURE 2. Examples of repetitive stereotyped polygraphic patterns occurring in three patients are represented one for each line. a) Pattern 1: sudden appearance of a cluster of significantly increased respiratory amplitude compared to the background, associated with bradypnoea and central apnoea, intense and transient activation of the microphone, similar to a vocalisation, heart rate acceleration, limb movement and body position change; b) Pattern 2: sudden appearance of a cluster of breaths with irregular (varying) amplitude followed by a noise signal on the snoring microphone similar to a vocalisation, heart rate acceleration, limb movement and body position change; $\mathrm{c}$ ) Pattern 3: mixture of patterns 1 and 2. All three patterns are incompatible with breathing patterns in sleep or relaxed wakefulness [9, 10]. Micro: microphone; Flow: airflow; Tor: thoracic effort; Add: abdominal effort; $\mathrm{Sp}, \mathrm{O}_{2}$ : arterial oxygen saturation measured by pulse oximetry; fC: cardiac frequency; Pos: body position (Eret: upright; De: right; Su: supine; Si: left; Pr: prone); PLMs: activity of the anterior tibialis muscles. 

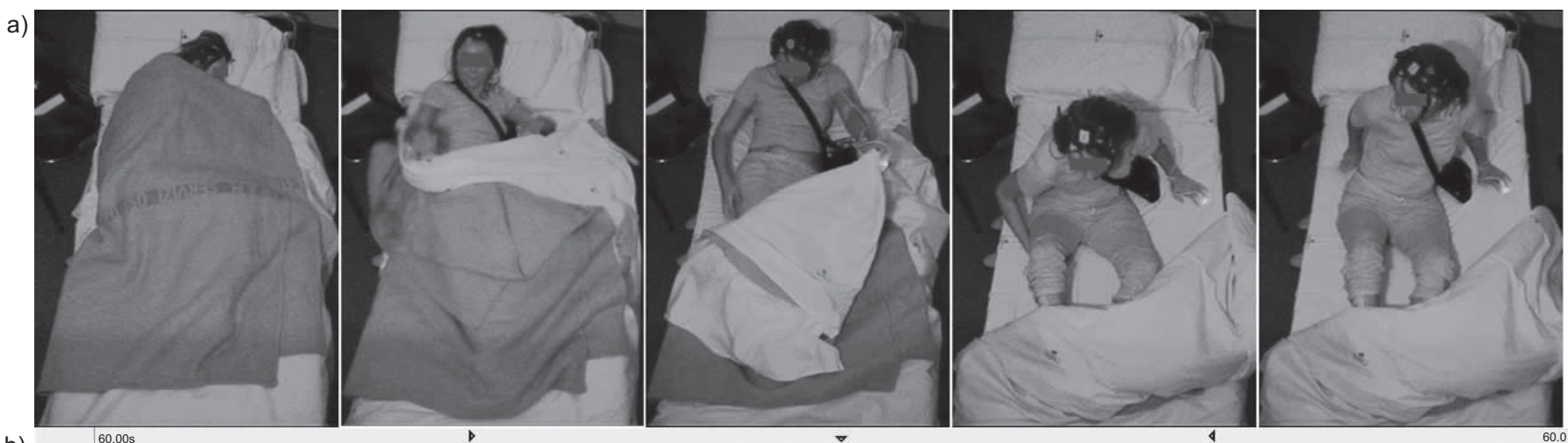

b)
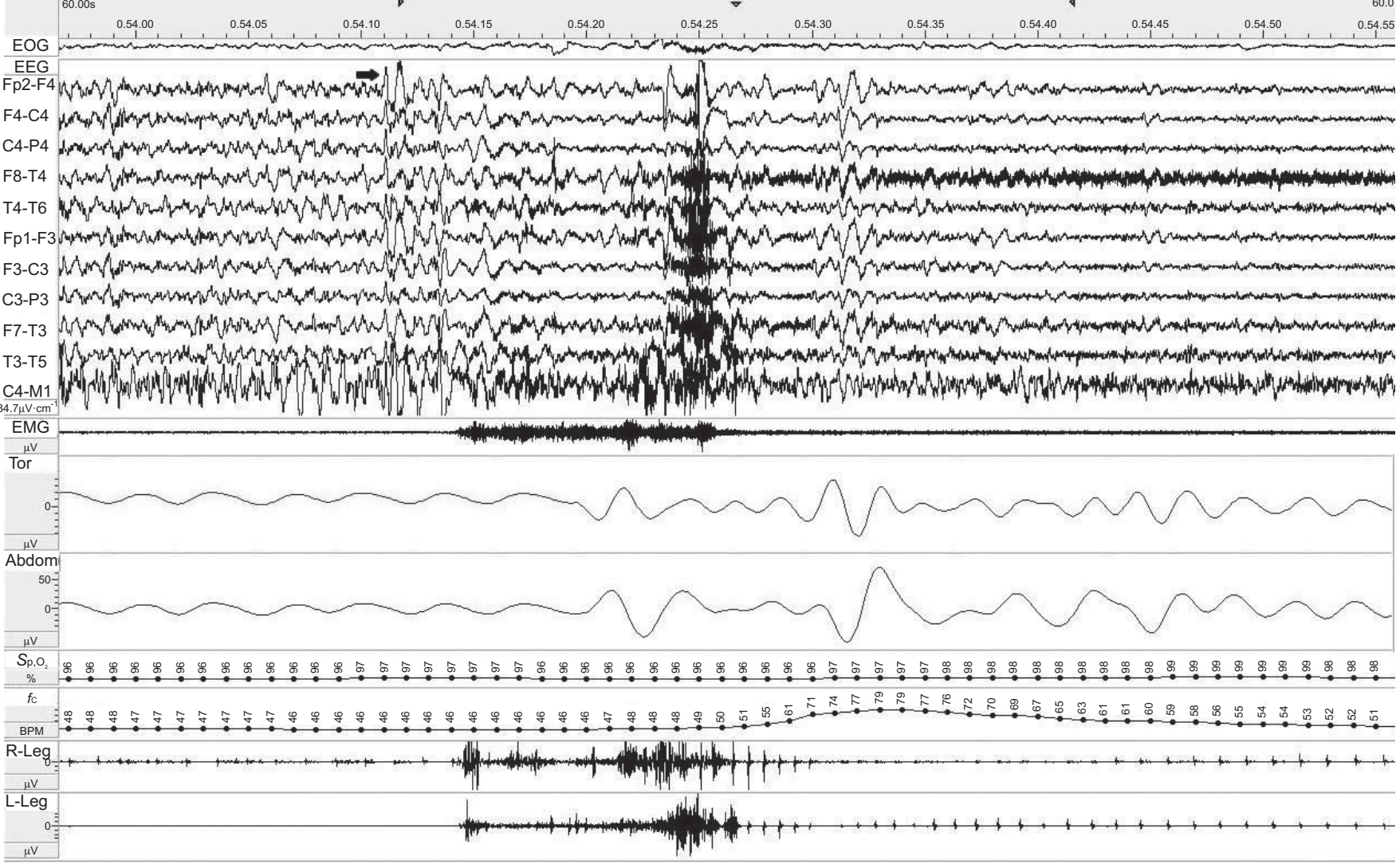

FIGURE 3. a) A video event of paroxysmal arousals characterised by abrupt awakening from sleep associated with confusional status. b) Polysomnography traces show that frontal epileptiform discharges (indicated by the arrow) triggered electroencephalographic arousals correlated with autonomic and muscle activation. EOG: electrooculographic channels; EEG: electroencephalographic channels; EMG: submental electromyogram; Tor: thoracic effort; Abdom: abdominal effort; $\mathrm{Sp}, \mathrm{O}_{2}$ : arterial oxygen saturation measured by pulse oximetry; fc: cardiac frequency; R-Leg: electromyogram of the right anterior tibialis muscles; L-Leg: electromyogram of the left anterior tibialis muscles.

and stereotyped events involving the limbs, the axial muscles and/or the head (fig. 3). Table 3 shows the spectrum of video PSG stereotyped movements ranging from MMEs to PAs and MAs.

The clinical history of nocturnal motor activity and the video PSG findings of at least one major attack (MA or PA) or at least two minor stereotyped episodes (MMEs) allowed us to diagnose NFLE in our patients. This diagnostic approach was confirmed by clinical improvement under drug treatment with carbamazepine. No evidence of cortical dysplasia or other parenchymal abnormalities emerged from magnetic resonance imaging analysis.

\section{DISCUSSION}

Typically performed in specialised sleep centres and attended by trained technologists, PSG remains the laboratory gold standard for the diagnosis of OSAS. However, in spite of its relevance, PSG is considered expensive and technically intense, and it has been argued that many patients do not require such a comprehensive procedure to diagnose uncomplicated OSAS [13].

The prevalence of OSAS and its association as an independent risk factor for motor vehicle accidents, hypertension and cardiovascular disease have made it a major public health issue. Although many private sleep laboratories have an 
acceptable patient waiting time for in-laboratory attended PSG, patient waiting times in public healthcare systems can exceed 6 months [14]. Pressure for alternative approaches to the inlaboratory management of patients with OSAS will continue to increase, given the cost of attended PSG, the limited number of laboratory facilities, and the growing clinical demand for more rapid access to testing. In this setting, demand for accurate and reliable portable monitors is growing [15]. Portable monitoring is widely used as an alternative approach to PSG in countries other than the USA. A position paper of the Canadian Sleep Society and Canadian Thoracic Society details their guidelines on the use of portable monitoring as a diagnostic tool for OSAS in adults and states that when used appropriately, portable monitoring may expedite treatment when there is a high clinical suspicion of OSAS [16]. In Italy, portable monitoring, performed most commonly in the patient's home, is increasingly being used clinically as an alternative to PSG to diagnose patients with uncomplicated OSAS. According to the Italian guidelines, in the presence of habitual snoring and awakenings with the feeling of choked gasps, a portable monitoring device can be used for the diagnosis of OSAS [17].

The present study suggests that portable monitor sensors should be applied to investigate not only SDB but that methodology should also include identification of stereotyped features which can suggest behavioural sleep-related abnormalities. PLM is a common finding in OSAS patients and sleep clinicians are able to recognise this pattern even in the absence of respiratory events. Investigation can become even more challenging when behavioural features lack a periodic distribution but still recur throughout the night with a clear stereotyped configuration (fig. 3). Paroxysmal behaviours, characterised by a rigid sequence of phenomena, but never heralded or followed by phasic respiratory events, may be suggestive of epileptic manifestations.

Despite the lack of gold-standard diagnosis of NFLE, the criteria adopted in the present study [18] lead to an unexpectedly high rate of NFLE diagnosis and maximum specificity of PSG recording.

This does not imply that all stereotyped patterns occurring during portable monitoring must be scored automatically as an ictal event. Without a consistent clinical history and video PSG evidence, the hypothesis of NFLE remains only a possible diagnosis. In addition, motor phenomena in NFLE are not always easily distinguishable from physiological sleep-related movements or non-rapid eye movement parasomnias [5]. In differential diagnosis between epileptic phenomena and nonepileptic behaviour occurring during sleep, topical features characterising nocturnal frontal seizures are several attacks per night at any time during the night and stereotyped motor patterns [19].

The relatively high number of female subjects in our sample is in contrast to the usual sex distribution in OSAS patients. This element, associated with a particular clinical history of nocturnal motor events, could anticipate the presence of atypical polygraphic patterns.

In any case, the clinical report of snoring and choking gasps recommends the use of portable monitoring as a first strategy.
In conclusion, portable monitoring for SDB diagnosis can disclose non-respiratory sleep disorders. In particular, when repetitive stereotyped features recur in a nocturnal portable monitoring, without evidence of respiratory events, a parasomnia or an epileptic manifestation should be suspected.

In our study, only recordings with $\mathrm{AHI}<5$ events $\cdot \mathrm{h}^{-1}$ were selected in order to investigate the stereotyped polygraphic patterns that were not justified by a diagnosis of SDB and to clarify the origin of the symptoms described by patients. Since NFLE and SDB can coexist and potentially exacerbate each other, further studies should investigate the presence of these atypical patterns in patients with OSAS.

A methodological weakness of the present study is based on the consideration that level 3 portable monitors do not record EEG, electro-oculogram, or chin muscle activity and therefore cannot detect whether the patient is awake or asleep during the recording. However, breathing flow features for the detection of wakefulness, previously described in patients with OSAS $[9,10]$, are different from the patterns described in our study, which were characterised by an atypical, repetitive and stereotyped morphology. In addition, the lack of EEG channels and video control in portable monitoring cannot allow scoring of EDs and their correlation with paroxysmal motor attacks. For this reason, identification of stereotyped and recurrent patterns during cardiorespiratory monitoring, in the absence of abnormal breathing features, should be explored with great attention, particularly in patients with non-restorative sleep. In these not infrequent cases, a nocturnal video PSG based on the new AASM scoring rules [10], which require frontal EEG derivation, is recommended for a reliable diagnosis of NFLE.

\section{STATEMENT OF INTEREST}

None declared.

\section{ACKNOWLEDGEMENTS}

We wish to thank N. Azzi, A. Abramo and I. Pollara (Sleep Disorders Centre, Dept of Neurosciences, University of Parma, Parma, Italy) for their valuable technical support.

\section{REFERENCES}

1 American Academy of Sleep Medicine. International Classification of Sleep Disorders. 2nd Edn. Westchester, AASM, 2005.

2 Sforza E, Montagna P, Rinaldi R, et al. Paroxysmal periodic motor attacks during sleep: clinical and polygraphic features. Electroenceph Clin Neurophysiol 1993; 86: 161-166.

3 Tinuper P, Lugaresi E. The concept of paroxysmal nocturnal dystonia. In: Bazil CW, Malow BA, Sammaritano MR, eds. Sleep and Epilepsy: the Clinical Spectrum. Amsterdam, Elsevier Science, 2002; pp. 277-282.

4 Provini F, Plazzi G, Tinuper P, et al. Nocturnal frontal lobe epilepsy. A clinical and polygraphic overview of 100 consecutive cases. Brain 1999; 122: 1017-1031.

5 Zucconi M, Ferini-Strambi L. NREM parasomnias: arousal disorders and differentiation from nocturnal frontal lobe epilepsy. Clin Neurophysiol 2000; 111: 129-135.

6 Oldani A, Zucconi M, Castronovo C, et al. Nocturnal frontal lobe epilepsy misdiagnosed as sleep apnea syndrome. Acta Neurol Scand 1998; 98: 67-71.

7 Ferber R, Millman R, Coppola M, et al. Portable recording in the assessment of obstructive sleep apnea: ASDA standards of practice. Sleep 1994; 17: 378-392. 
8 Iber C, Ancoli-Israel, Chesson A, et al. The AASM Manual for the Scoring of Sleep and Associated Events. Rules, Terminology and Technical Specifications. Westchester, AASM, 2007.

9 Guerrero A, Embid C, Farre R, et al. Sleep breathing flow characteristics as a sign for the detection of wakefulness in patients with sleep apnea. Respiration 2010; 80: 495-499.

10 Ayappa I, Norman RG, Whiting D, et al. Irregular respiration as a marker of wakefulness during titration of CPAP. Sleep 2009; 32: 99-104.

11 Terzano MG, Parrino L, Smerieri A, et al. Atlas, rules and recording techniques for the scoring of cyclic alternating pattern (CAP) in human sleep. Sleep Med 2001; 2: 537-553.

12 Johns MW. A new method for measuring daytime sleepiness: The Epworth Sleepiness Scale. Sleep 1991; 14: 540-545.

13 Collop NA. Portable monitoring for the diagnosis of obstructive sleep apnea. Curr Opin Pulm Med 2008; 14: 525-529.

14 Kuna ST. Portable-monitor testing: an alternative strategy for managing patients with obstructive sleep apnea. Respir Care 2010; 55: 1196-1215.
15 McNicholas WT, Levy P. Portable monitoring in sleep apnoea: the way forward? Eur Respir J 2011; 37: 749-751.

16 Blackman A, McGregor C, Dales R. et al. Canadian Sleep Society/ Canadian Thoracic Society position paper on the use of portable monitoring for the diagnosis of obstructive sleep apnea/hypopnea in adults. Can Respir J 2010; 17: 229-232.

17 Commissione Paritetica Associazione Italiana Medicina del Sonno (AIMS), Associazione Italiana Pneumologi Ospedalieri (AIPO). Linee guida di procedura diagnostica nella sindrome delle apnee ostruttive nel sonno dell'adulto. [Guidelines for diagnostic procedure in the obstructive sleep apnoea syndrome in adults.]. Med Sonno 2000; 3: 2-6.

18 Bisulli F, Vignatelli L, Naldi I, et al. Diagnostic accuracy of a structured interview for nocturnal frontal lobe epilepsy (SINFLE): a proposal for developing diagnostic criteria. Sleep Med 2012; 13: 81-87.

19 Tinuper P, Provini F, Bisulli F, et al. Movement disorders in sleep: guidelines for differentiating epileptic from non-epileptic motor phenomena arising from sleep. Sleep Med Rev 2007; 11: 255-267. 